\title{
Adaptive Neuro-Fuzzy control of Autonomous Ground Vehicle (AGV) based on Machine Vision
}

\author{
Hassan Farahat', Sameh Farid ${ }^{r}$, Osama E. Mahmoud ${ }^{r}$
}

I Pyramids Higher Institute (PHI), Giza, Egypt.
rFaculty of Engineering, MSA University, Giza, Egypt.
rFaculty of Engineering-Mattaria, Helwan University, Helwan, Egypt
Emails: Hassan.elkholy`@ @mail.com; dr.sam.far@gmail.com; osama_ismail@m-eng.helwan.edu.eg

\begin{abstract}
Development of self-autonomous robot that has the capability to deal with dynamic obstacles as well as static obstacles and keep finding its optimal path represent big challenge to many of the researchers nowadays. Utilization of artificial intelligence (AI) in developing a robust algorithm for nonlinear system was proved in some applications. The present work focus on excessive analysis and investigation of the optimal AI algorithm technique for controlling the path planning of four -wheel autonomous robot to achieve its set target through both static and dynamic obstacles with the optimal path without human interference. Fuzzy logic, Neural Network, and Adaptive Neuro-Fuzzy Inference System (ANFIS) were tested and evaluated as controlling algorithm for this navigation task. The performance evaluation was done both theoretically and experimentally using vision-based feedback sensor, Kinect camera, on a real four-wheel mobile robot. Results prove that ANFIS successfully control the automatic path planning of the robot with the optimal path by reaching its target with $1 \cdot . \wedge \varepsilon \%$ less than Neural Network and 10. $40 \%$ less than Fuzzy Logic. ANFIS controller also has given safer path as well as flexible obstacles avoidance through its path to final destination with smooth time-efficient path in the cluttered environment.
\end{abstract}

Keywords: Autonomous robots, Machine Vision, Artificial intelligence, Path planning, $\quad$ ANFIS, cluttered Environments 


\section{Introduction and Problem Statement}

Self-autonomous robot has a great interest in computer science and artificial intelligence due to its speed, efficiency, avoiding of static and dynamic obstacles, changing paths based on visual perception of its environment and the autonomous robot should be able to detect the shortest path to reach the target [']. Navigation and obstacle avoidance are one of the fundamental problems in mobile robotics, which the researchers have been working on solving this problems in the past two decades $[r]$. Presently, Neural Network integrated Fuzzy logic, which integrates the fuzzy logic representation of human knowledge with the learning capability of neural networks, to solve nonlinear system dynamic control problems [ $\left.{ }^{\top}\right]$. The main drawback of fuzzy logic is the lack of a systematic methodology for their design. Usually, tuning parameters of membership functions is a time-consuming task. Neural network learning techniques can automate this process, significantly reducing development time, and resulting in better performance. The merger of neural networks and fuzzy logic led to the creation of neuro-fuzzy technique which is currently one of the most research fields [ ${ }^{\varepsilon}$ ]. The ANFIS approach is becoming one of the major areas of interest because it gets the benefits of neural networks as well as of fuzzy logic systems $\left[{ }^{0}, 7\right]$. For real time autonomous navigation of the wheeled autonomous robot should be capable of sensing its environment, interpreting the sensed information to obtain the knowledge of its position and the environment, planning a real-time route from an initial position to a target with obstacle avoidance $[\vee, \wedge, 9]$. Car-like robot with the integration of front-wheel driving and steering system has been implemented and simulated. Also, the robot was designed not only to avoid the jamming in the motion, but also to owe the advantages of compacter structure, lighter body, and lower energy consumption $[1 \cdot, 11]$. Increasing the speed and accuracy of autonomous robots are the main issues to be addressed for generalization of autonomous robotics systems. Simulation software and virtual environments are the two potential tools for accelerating the design and development of autonomous robots. For this, V-REP and LabVIEW have been used in order to optimize the (AI) algorithm design in cluttered environments $[1 \leqslant, 10]$. In this paper ANFIS approach has been analyzed for real time navigation of autonomous robots, to determine optimal path of wheeled autonomous robot navigating in unknown and cluttered environment. Simulation examples are presented to test and evaluate the most suitable technique, then to validate this experimentally.

\section{r Methodology and the Proposed System Description}

In order to achieve the optimal path navigation model, the akerman (Four-wheel autonomous robot) has been modeled in $[1 Y]$ that is suitable for simulation experiments. It requires to take into account various physical, mathematical or technological characteristics to define this kind of autonomous robot[ $\left.{ }^{1}\right\}$. The akeramn 
driven four-wheel chassis concept that have wheels placed in the same axis of rotation is a popular choice because of its mobility in plane and simplicity. This type of autonomous robot has been designed using solidwork and implemented in the Experimental work as shown in fig. 1 . The vehicle system is the experimental platform that consists of a miniature car in scale 1:17. It is equipped with brushed DC motor through a gear box for driving the four wheels and servo motor for two front steering wheels. And four-wheel double wishbone oil filled aluminum capped shocks. It is still conveniently small for driving indoors in order to use the car indoors without risking crashes. The chassis of the robot measures $80 \times 50 \mathrm{~cm}(\mathrm{D} \times \mathrm{H})$, weight $\wedge \mathrm{kg}$. It is equipped with DC brushed servo motors with incremental encoders and Kinect sensor and the sensors with range $0.5-4$ meters. The speed range is $0-0.5 \mathrm{~m} / \mathrm{s}$, power bank and Lithium-Polymer battery pack $(r \cdots \mathrm{mAh})$ is used for powering the autonomous robot as shown in Fig. .

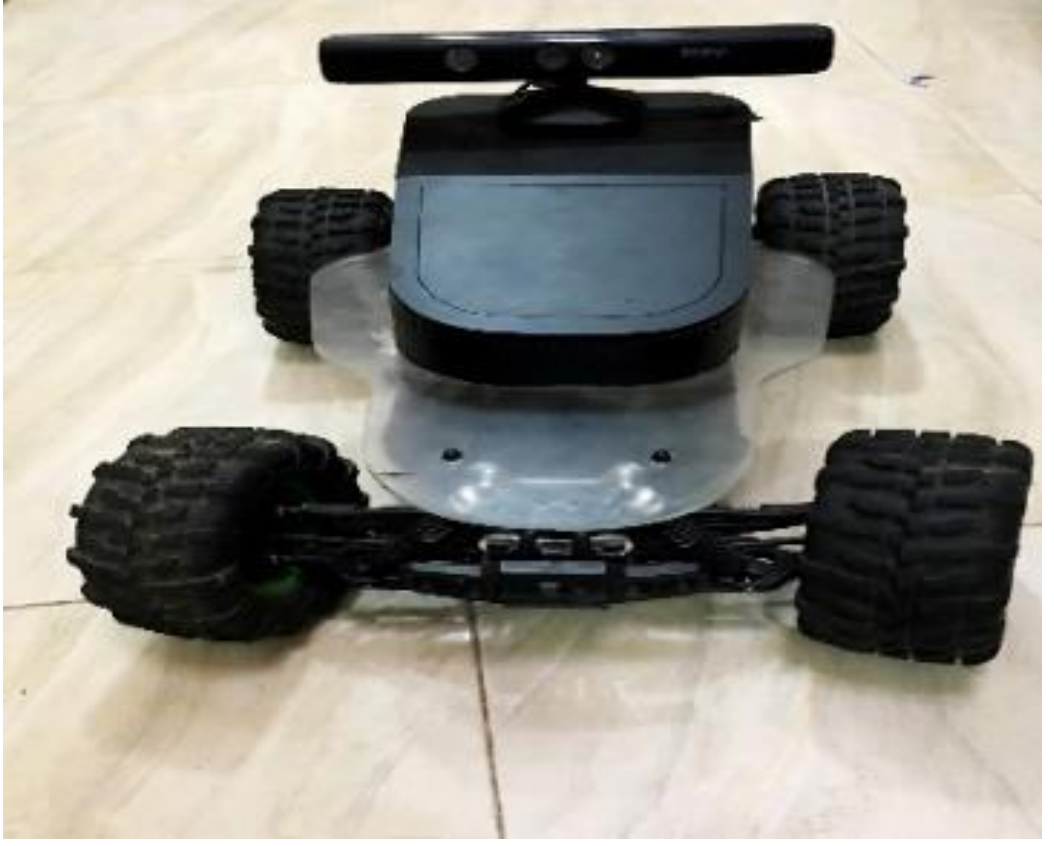

Fig. '. The proposed hardware self-autonomous robot

Easily evaluate how your product will perform and move throughout its operational cycle with motion analysis using SOLIDWORKS Simulation. Tightly integrated with SOLIDWORKS CAD, motion analysis using SOLIDWORKS Simulation a regular part of design process reducing the need for costly prototypes, eliminating rework or delays, and saving time and development costs. Car-like robot consist of one fixed wheel at the back and one steerable wheel in the front. The generalized coordinates for this type of robot are $\mathrm{q}=(\mathrm{x}, \mathrm{y}, \theta, \varphi)$ where $(\mathrm{x}, \mathrm{y})$ are the Cartesian coordinates of the rear axle midpoint, $\theta$ represents the orientation of the car with respect to the $\mathrm{x}$-axis and $\varphi$ is the steering angle, as presented in Fig. ${ }^{r}$. 


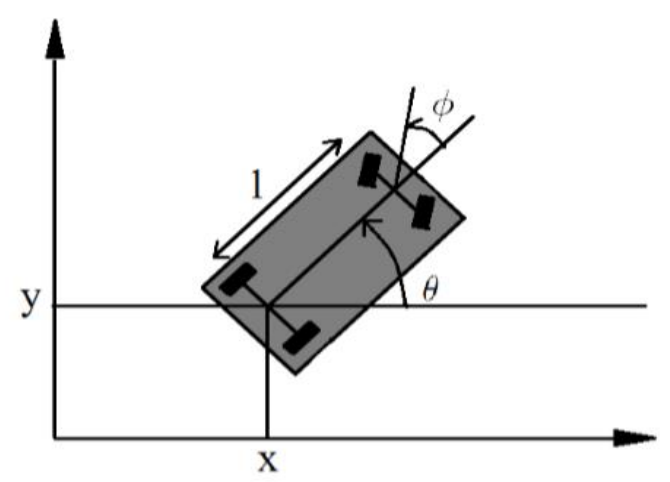

Fig. ${ }^{\Upsilon}$. Schematic of a car-like robot platform

The kinematic model of this kind of mobile robot described by the following nonlinear equation:

$$
\begin{aligned}
\dot{x} & =v \cdot \cos (\theta) \\
\dot{y} & =v \cdot \sin (\theta) \\
\dot{\theta} & =\omega=v \cdot \frac{\tan (\varnothing)}{L}
\end{aligned}
$$

where $\mathrm{L}$ is the distance between the front and rear axles

The motion of the system is restricted by two nonholonomic constraints, one for each wheel

$$
\begin{gathered}
\dot{x}_{f} \sin (\theta+\emptyset)-\dot{y}_{f} \cos (\theta+\emptyset)=\cdot \\
\dot{x} \sin \theta-\dot{y} \cos \theta=.
\end{gathered}
$$

where $\left(x_{f}, y_{f}\right)$ are the coordinates of the midpoint on the front axle. With the use of the rigid-body constraint:

$$
\begin{array}{r}
x_{f}=x+L \cos \theta \\
y_{f}=y+L \sin \theta
\end{array}
$$

The first kinematic constraint then becomes:

$$
\dot{x} \sin (\theta+\emptyset)-\dot{y} \cos (\theta+\emptyset)-\dot{\theta} L \cos \dot{\emptyset}=.
$$


The allowed general velocities can be described by:

$$
\left[\begin{array}{c}
\dot{x} \\
\dot{y} \\
\dot{\theta} \\
\dot{\phi}
\end{array}\right]=\left[\begin{array}{c}
\cos \theta \cos \emptyset \\
\sin \theta \sin \emptyset \\
\frac{\sin \emptyset}{L}
\end{array}\right] u_{1}+\left[\begin{array}{l}
\cdot \\
\cdot \\
1
\end{array}\right] u_{r}
$$

where $u$, the driving velocity input and $u_{r}$ the steering velocity input. The steering velocity of the front wheels, switches back and forth more.

The system limitation of the steering angle range is from - $\varepsilon \cdot$ to $\varepsilon \cdot$ and the Kinect sensor range is $0.5-4$ meters. The simplistic implementation of the real-life scenarios where cameras are used to capture different parts of the entire workspace and their outputs are fused to create an overall map used by the optimal path planning algorithm technique. Kinect sensor has been used in the front of the robot for the image capturing and real time path creation as shown in Fig. ${ }^{\top}$. The map of environment represents as a grid of cells (pixels) cell $_{i}=\left\{x_{i}, y_{i, F_{i}}\right\}$ where each cell has a triple of coordinates $\mathrm{x}, \mathrm{y}$ and an information value $\mathrm{F}$. According to this map, the image is represented as a grid of integer values from top- bottom and left-right. $\mathrm{F}$ is a Boolean variable FALSE (•) or TRUE ( 1 ), and denotes if some obstacle or part of it is within that cell or not. In case of "TRUE" value the cell is filled, otherwise the cell is empty.

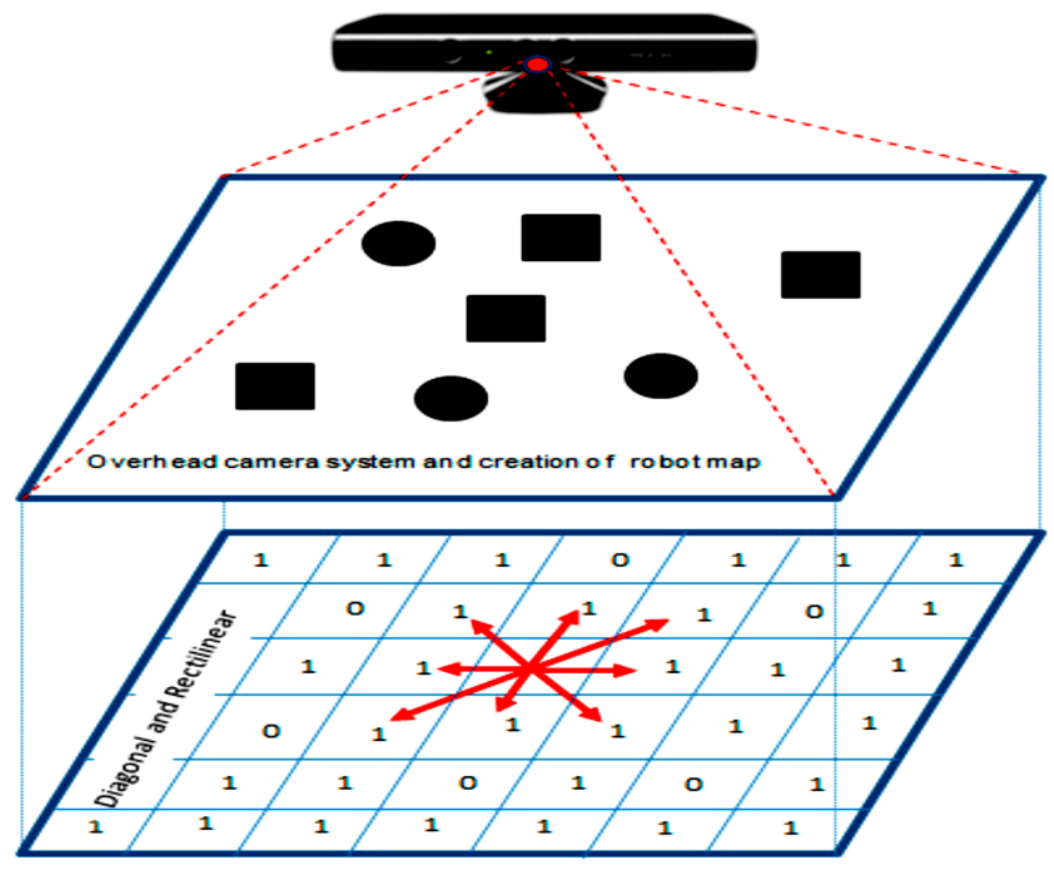

Fig. r. Kinect camera creation robot map $[1 r]$. 


\section{r Path Planning and ANFIS Controller Algorithm}

The adaptive neuro fuzzy inference system (ANFIS) is an integrated system of artificial neural network (ANN) and fuzzy inference system (FIS). The ANFIS controller for the wheeled autonomous robot navigation can be easily implemented for real-time applications because this controller handles unknown and uncertain situations with the simple computations. The proposed navigation controller working is based on the local information collected from the Kinect sensor. The ANFIS analyzed here is a first order Takagi Sugeno Fuzzy Model, In the current analysis there are four inputs: Front obstacle distance $x_{1}$, Right obstacle distance $x_{\uparrow}$,Left obstacle distance $x_{\uparrow}$ and Target angle $x_{\varepsilon}$ and the output is the Steering angle. The if-then rules for the ANFIS architecture are defined as follows:

$$
\begin{aligned}
& \text { Rule: IF } x_{1} \text { is } A_{j} ; x_{2} \text { is } B_{k} ; x_{3} \text { is } C_{m} ; x_{4} \text { is } D_{n} \text { THEN } F_{i}=p_{i} x_{1}+r_{i} x_{2}+s_{i} x_{3}+t_{i} x_{4}+u_{i} \\
& \text { where } j=1 \text { to } q_{1} ; k=1 \text { to } q_{2} ; m=1 \text { to } q_{r} ; n=1 \text { to } q_{4} \text { and } i=1 \text { to } q_{1} \cdot q_{2} \cdot q_{3} \cdot q_{4}
\end{aligned}
$$

$\mathrm{A}, \mathrm{B}, \mathrm{C}, \mathrm{D}$ are the fuzzy membership sets defined for the input variables $x_{1}, x_{r}, x_{r}, x_{\varepsilon}$. $q_{1}, q_{r}, q_{r}, q_{\varepsilon}$ are the number of membership functions for the fuzzy systems of the inputs $x_{1}, x_{\uparrow}, x_{r}, x_{\varepsilon}$ respectively. $F_{i}$ are the linear consequent functions defined in terms of the inputs $\left(x_{1}, x_{\uparrow}, x_{\uparrow}, x_{\varepsilon}\right) . p_{i}, r_{i}, s_{i}, t_{i}, u_{i}$ are the consequent parameters of the ANFIS fuzzy model. In the ANFIS model, nodes of the same layer have similar functions. The output signals from the nodes of the previous layer are the input signals for the current layer. The output obtained with the help of the node function will be the input signals for the subsequent layer as shown in Fig. $\{$.

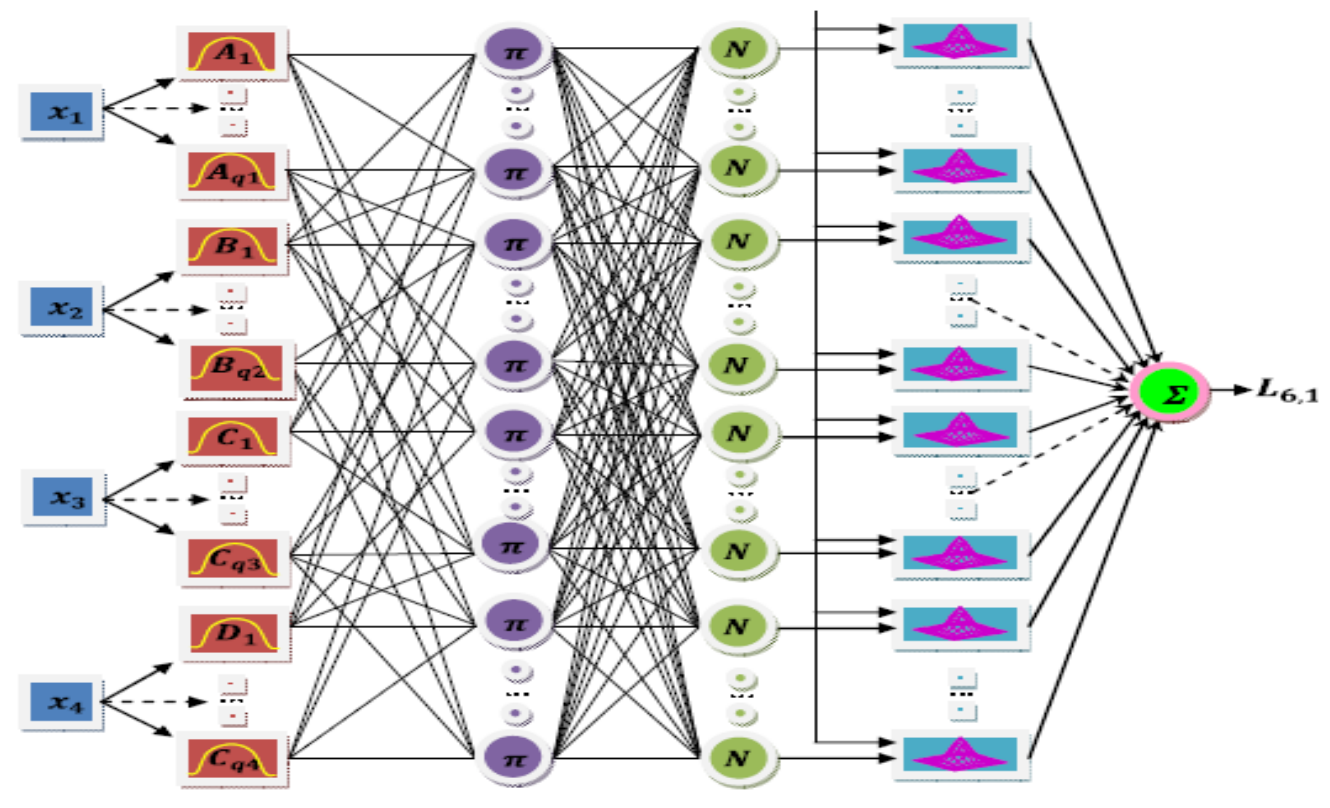

Fig. ¿. Six-layer ANFIS for robot navigation 
Layer 1: The input layer receives signal from arrays of sensors $x_{1}, x_{\uparrow}, x_{\uparrow}, x_{\varepsilon}$. which defines the static as well as moving obstacles and target poisons from the target tracker robot.

Layer $r$ : Every node in this layer is an adaptive node (square node) with a particular fuzzy membership function (node function) specifying the degrees to which the inputs satisfy the quantifier. For four inputs the outputs from nodes are given as follows;

$$
\begin{aligned}
& L_{\ulcorner g}=\mu_{A g}(x) \text { for } g=1, \ldots, q \text {, } \\
& \text { (for input } x_{1} \text { ) } \\
& L_{r g}=\mu_{B g}(x) \text { for } g=q_{1}+1, \ldots, q_{1}+q_{r} \\
& L_{r g}=\mu_{C g}(x) \text { for } g=q_{1}+q_{r}+1, \ldots, q_{1}+q_{r}+q_{r} \\
& \text { (for input } x_{Y} \text { ) } \\
& L_{r g}=\mu_{D g}(x) \text { for } g=q_{1}+q_{r}+q_{r}+1, \ldots, q_{1}+q_{r}+q_{r}+q_{\varepsilon} \text { (for input } x_{\varepsilon} \text { ) }
\end{aligned}
$$

Here the membership function for left obstacles (A), right obstacles (B), front obstacles (C), and target angle(D). The structure of ANFIS controller has two membership functions for all inputs, i.e. front, right and left obstacles and target steering as shown in Fig. ₹. The linguistic variables of the left-obs are: Very Very Far: VVF, Very Far: VF, Far: F, Medium: M, Near: N, Very Near: VN, and Very Very Near: VVN. The linguistic variables of the tar-ang are: Very Very Positive: VVP, Very Positive: VP, Positive:P, Zero: Z, Near Negative: N, Very Negative: VN, and Very Very Negative: VVN.
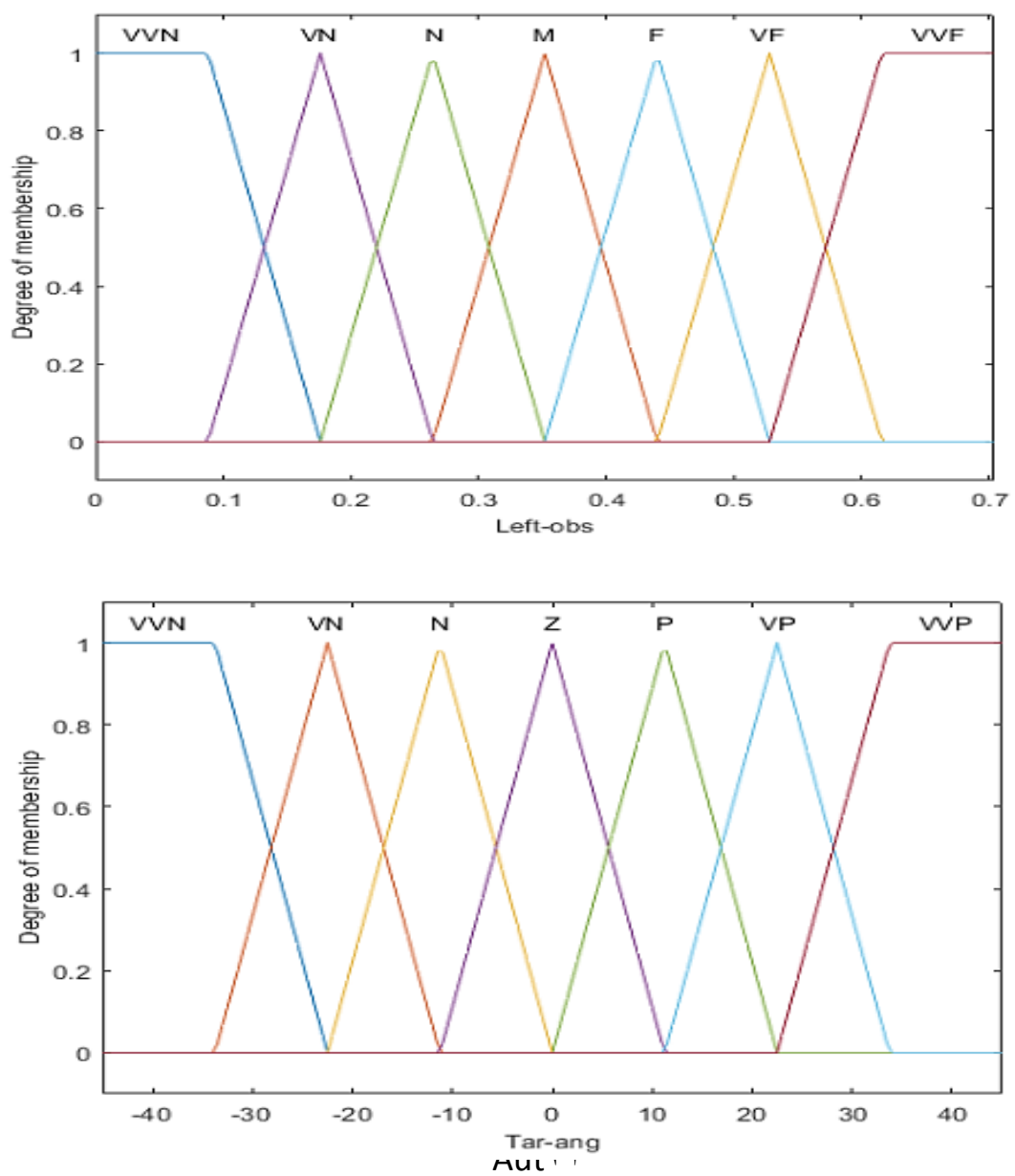


\section{Fig. ‥ Inputs Fuzzy memberships of ANFIS control system.}

Layer ${ }^{r}$ : Every node in this layer is a fixed node (circular) labeled as ' $\pi$ '. The output denoted by $L_{\curlyvee i}$ the output is the product of all incoming signal.

$$
L_{{ }^{\prime} i}=W_{i}=\mu_{A g}(x), \mu_{B g}(x), \mu_{C g}(x), \mu_{D g}(x)
$$

For $i=1, \ldots ., q_{1} . q_{r} . q_{r} . q_{\varepsilon}$ and $g=1, \ldots ., q_{1}+q_{r}+q_{r}+q_{\varepsilon}$

The output of each node of the second layer represents the firing strength (degree of fulfillment) of the associated rule.

Layer : : Every node in this layer is a fixed node (circular) labeled as "N". The output of the $i^{\text {th }}$ node is calculated by taking the ratio of firing strength of $i^{t h}$ rule $W_{i}$ to the sum of all rules' firing strength.

$$
L_{4 i}=\bar{W}_{i}=\frac{W_{i}}{\sum_{r=1}^{r=q_{1} \cdot q_{2} \cdot q_{3} \cdot q_{4}} W_{r}} \text { This output gives a normalized firing strength. }
$$

Layer $\bullet:$ : Every node in this layer is an adaptive node (square node) whose output is simply the product of the normalized firing strength. A defuzzification node determines the weighted consequent value of a given rule. The connection between the inputs and output of this layer can be expressed as follows:

$$
L_{5 i}=\bar{W}_{i} f_{i}=\bar{W}_{i}\left(p_{i} x_{1}+r_{i} x_{2}+s_{i} x_{3}+t_{i} x_{4}+u_{i}\right)
$$

Where $\bar{W}_{i}$ is a normalized firing strength form (output) from layer $r$ and $\left\{p_{i}, r_{i}, s_{i}, t_{i}, u_{i}\right\}$ is the parameter set for steering angle. Parameters in this layer are referred to as consequent parameters.

Layer ": The single node in this layer is a fixed node (circular) labeled as " $\Sigma$ ", which computes the sum of the outputs of all defuzzification nodes and gives the overall system output that is steering angle as given below:

$$
L_{6 i}=\sum_{r=1}^{r=q_{1} \cdot q_{2} \cdot q_{3} \cdot q_{4}} \overline{W_{i}} f_{i}=\frac{\sum_{i=1}^{i=q_{1} \cdot q_{2} \cdot q_{3} \cdot q_{4}} W_{i} f_{i}}{\sum_{i=1}^{i=q_{1} \cdot q_{2} \cdot q_{3} \cdot q_{4}} W_{i}}
$$

The inputs of fuzzy logic layer are the front obstacle distance, left obstacle distance, right obstacle distance and target steering. A learning algorithm based on Neural Network technique has been developed to tune the parameters of Fuzzy membership functions, which has a smooth path generated by the Fuzzy Logic system. Using the developed ANFIS controller, the wheeled autonomous robots are able to avoid static and dynamic obstacles, and reach the target successfully in cluttered environments. 
ANFIS completed all the training and the generation of rules, the r-D plots, which show the relationship between the inputs and the output in Fig. ${ }^{7}$. The training results, used mean squared error of the angular velocity for obstacle avoidance behavior. The best validation performance is $\cdot .09 .79$ at apoch $\Lambda$. in Fig.. .
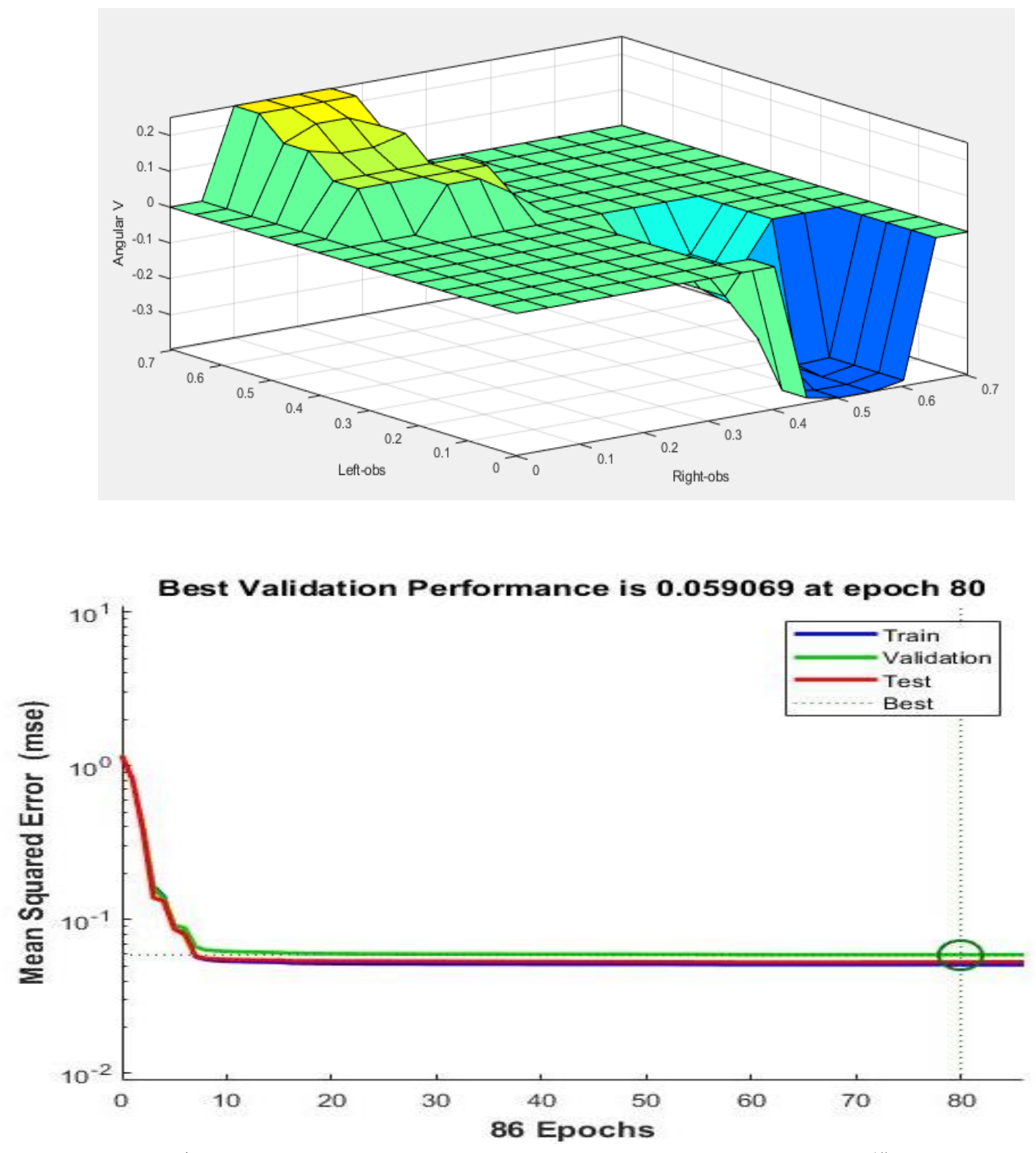

Fig.. . The surface view for obstacle avoidance ANFIS in ${ }^{\top} D$

Fig. ${ }^{\vee}$. Performance plot for training ANFIS 


\section{$\varepsilon \quad$ Simulation Results and Discussion}

The simulation results are obtained by using v-rep software and LABVIEW which have been developed in the laboratory using MATLAB. Fig.^ shows a typical screen of the software to demonstrate the effectiveness and the robustness of the ANFIS system, simulation results on autonomous robot by Artificial intelligence (AI) algorithms such as Fuzzy Logic, Neural Network and Adaptive neuro-fuzzy inference system (ANFIS) are exhibited. Autonomous robot should be able to identify feature, detect obstacles, target, learn from experience and find optimal path. The obstacle avoidance behavior is activated when the reading from Kinect camera detect obstacles. When an obstacle is detected too close to the autonomous robot, it avoids a collision by moving away from it in the opposite direction. Collision avoidance has the highest priority and therefore, it can override other behaviors; in this case, its main reactive behavior is decelerating for static as well as dynamic obstacle avoidance, find the optimal path by reaching its target as shown in Fig.^. Autonomous robot path is planned with different obstacles position by ANFIS algorithm technique as shown in Fig. 9. It is found that the navigation of autonomous robot with purposed ANFIS method has better performance than the fuzzy as well as neural controller in terms of positioning accuracy, collision avoidance and optimal path.

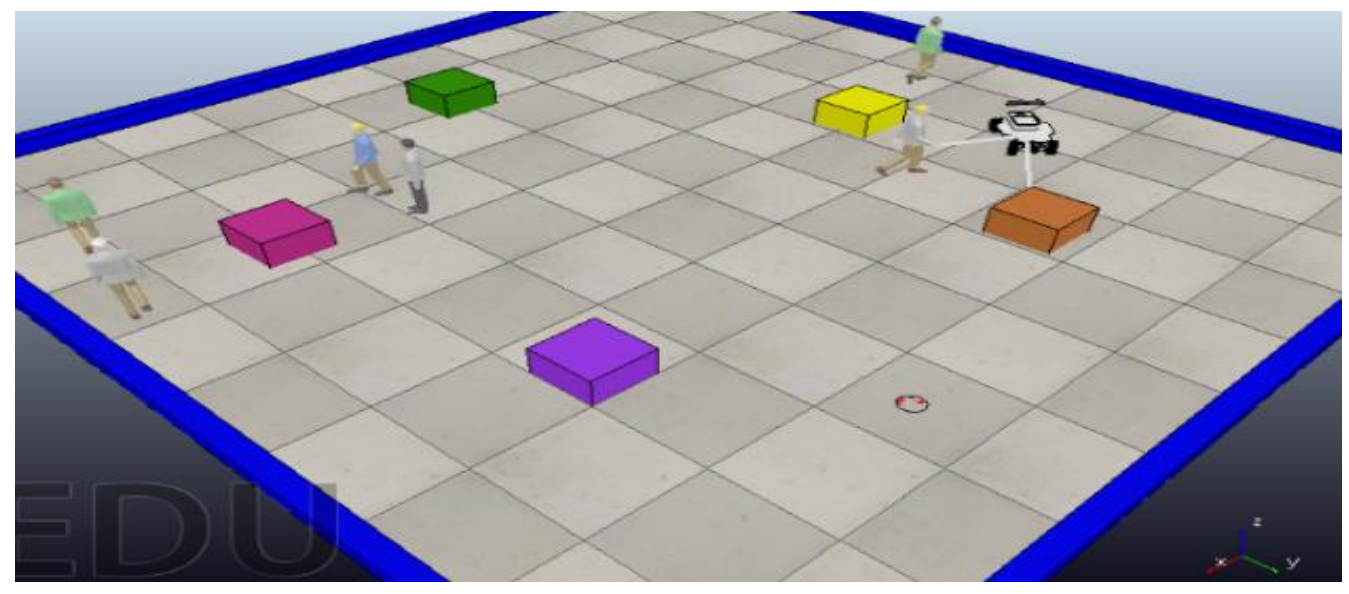

Fig. ^. V-REP and MATLAB simulation Environment

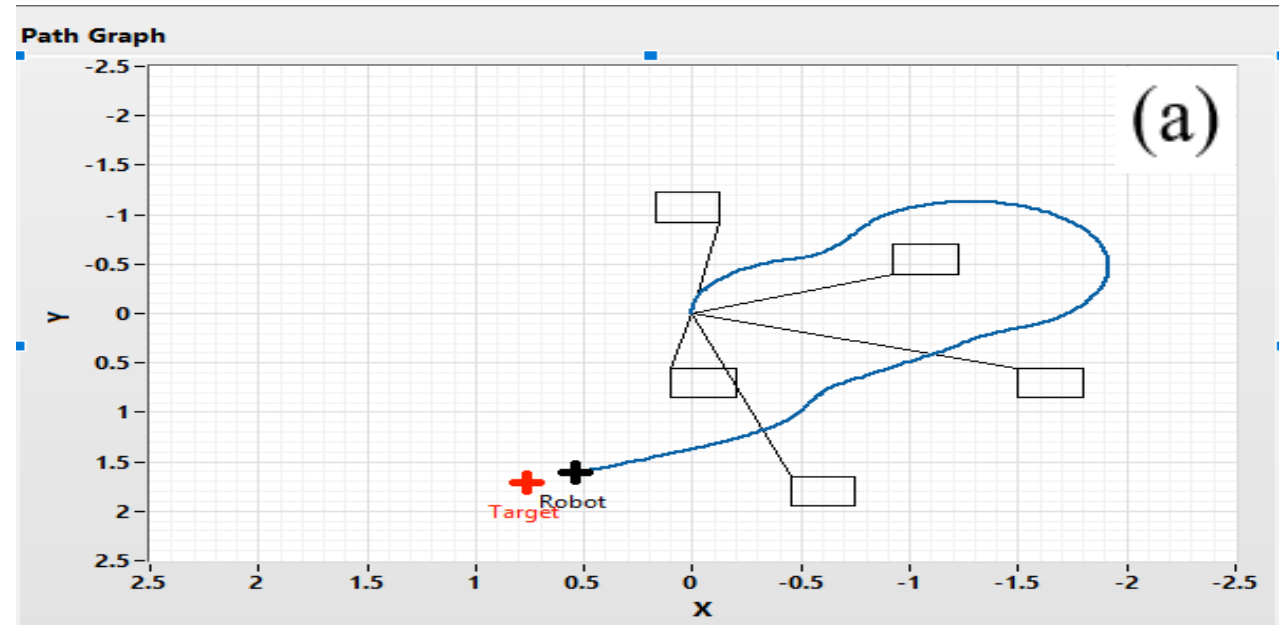




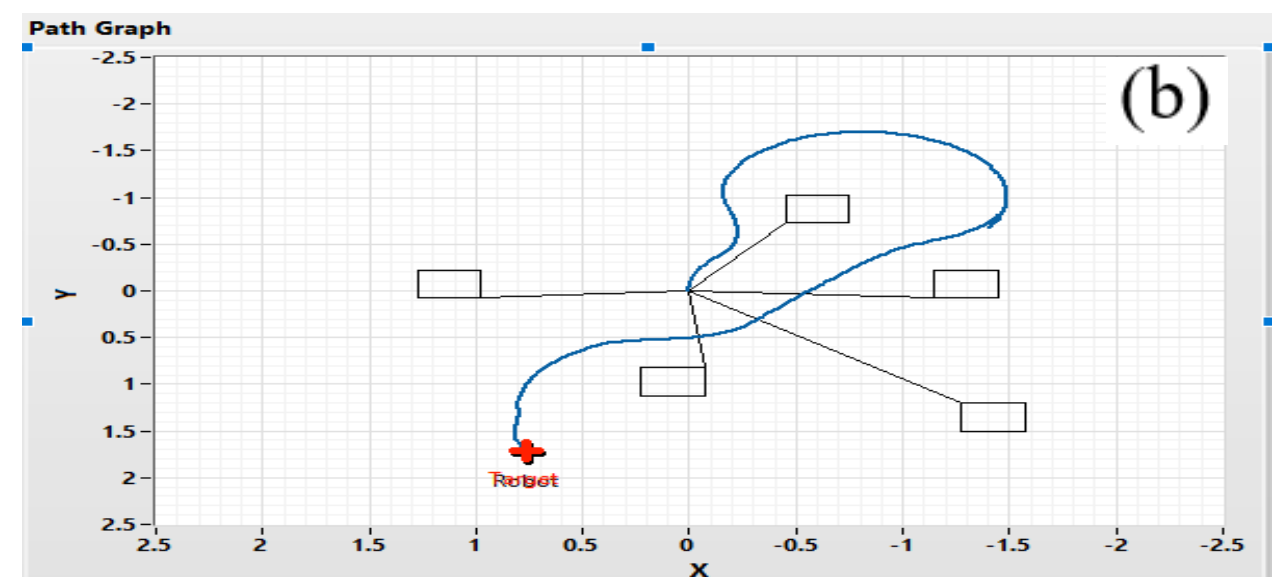

Fig. १. Scenarios of paths to Simulation environments

\section{- Experimental Results}

The experimental results have been made into two main parts: firstly, hardware validation between simulation enticements and real hardware; secondly, compression between different path planning algorithms and the proposed ANFIS algorithm.

\section{0.) Hardware Validation}

The autonomous robot behavior is verified in simulation and experimental task by controlling the path of a four-wheel autonomous robot to achieve its set target through avoidance of both static and dynamic obstacles with the shortest path. Table I shows the length of path and time taken by the autonomous robot in simulation and in the experimental test scenario during target finding. Fig. 1. shows the hardware experimental result using ANFIS controller and that the result is recorded to compare it with the simulation result. It is observed that the autonomous robot is able to reach the target efficiently during simulation and experiment. However, there is an error ratio $7 . \vee \vee \%$ of the path's length of the target and an error rate $\vee .50 \%$ of arrival time of the target between simulation and experiment due to several factors, such as friction between mechanical parts and friction with the ground, which led to the effect on speed taking into consideration at the time of decision making.

Table '. Comparison between Real and Simulation Environment results from ANFIS

Observations

Simulation Experimental Error\%




\begin{tabular}{|c|c|c|c|}
\hline Length of path (m) & 0.9 & $7 . r$ & $7 . \vee V \%$ \\
\hline Time taken (sec) & 71 & Vr & V.ro\% \\
\hline
\end{tabular}

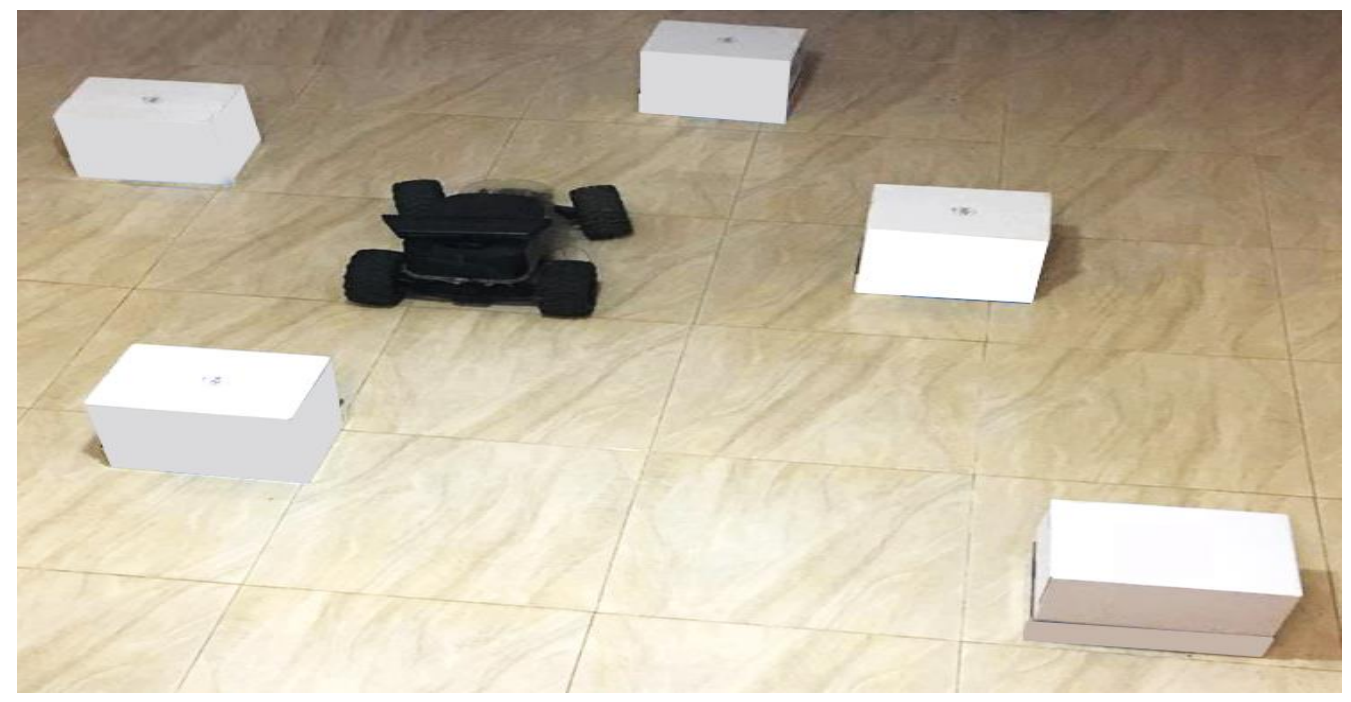

Fig. $1 \cdot$. Real Hardware Environment of autonomous robot

\section{- r Comparisons Between Path Planning Algorithms}

This part focuses on investigation of the optimal artificial intelligence algorithm for controlling the path of autonomous robot to achieve its set target .The algorithms techniques are Fuzzy logic, Neural Network, and Adaptive Neuro-Fuzzy Inference System (ANFIS), ANFIS (inputs: left, front, right obstacle distances and target angle) after learning, training and testing, autonomous robot gets the new steering angles. The autonomous robot only knows where it is and where the goal is. Furthermore, the optimality of a path, can be only determined after the completion of navigation, obtained by a concatenation of the paths to be decided upon at each instant of time. The solution obtained from current research is the navigational path analysis of autonomous robot in various environments. The results obtained from various approaches are given in Table $r$. It shows the deviation of experimental results with respect to simulation result in various controllers being used for finding the navigational optimal path and time taken to reach the target by autonomous robot. Results proved that ANFIS successfully control the automatic path planning of the robot with the optimal path by reaching its target with $1 \cdot . \wedge \Sigma \%$ less than Neural Network and $10.50 \%$ less than Fuzzy Logic as shown in Fig. ''. 
Table r. Comparison between different Path planning algorithms

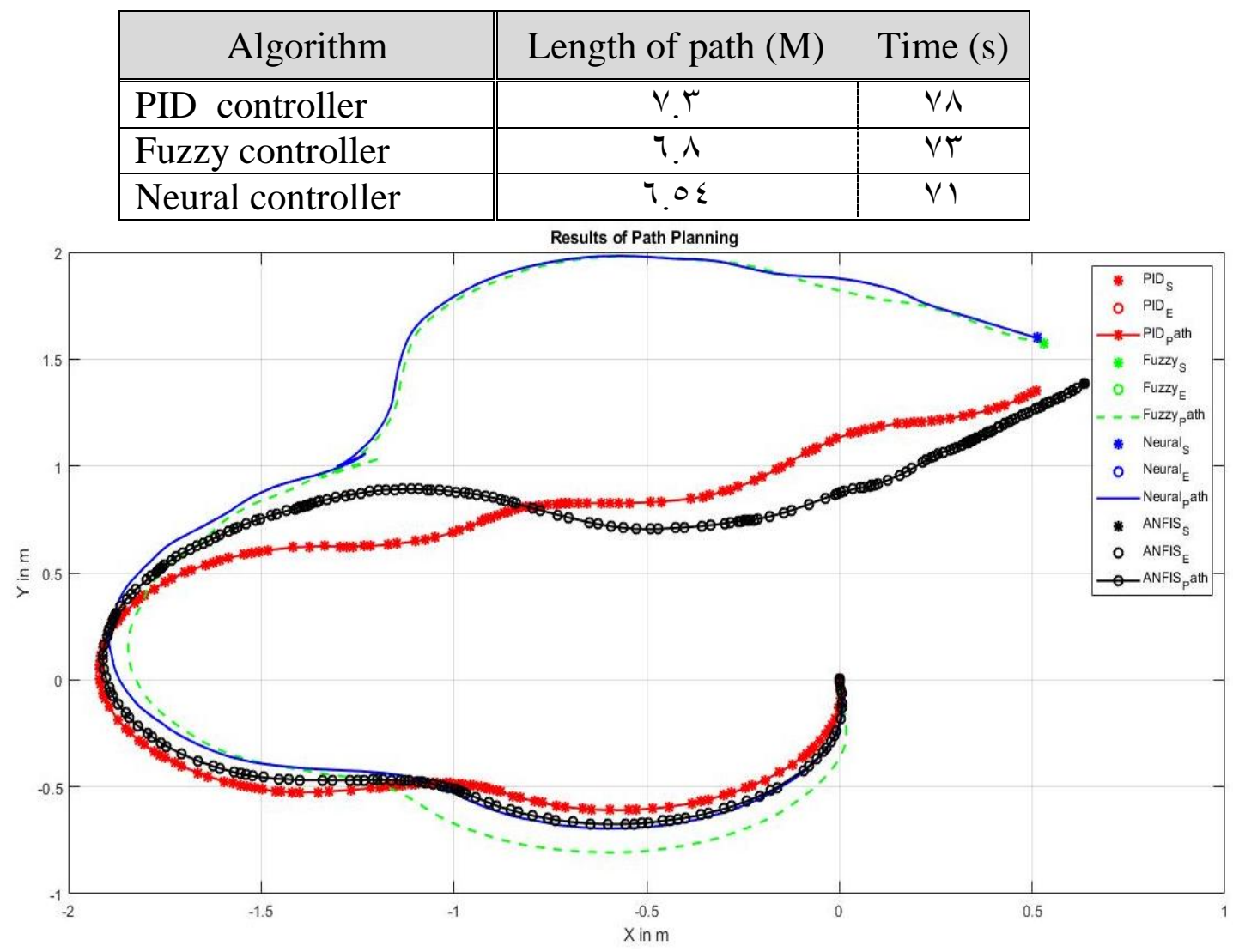

Fig. ' '. Experimental results using different path planning algorithm

\section{Conclusion}

Path planning in an unknown dynamic environment is one of the difficult challenges in autonomous robotics. The kinematic and dynamic analysis and modelling of the autonomous robot helps in giving the proper steering angle for the robot during the navigation. V-rep software has been used to handle the navigation control of autonomous robot in a simulation environment. Human pedestrian behavior to maintain and generate a safe and optimal path to avoid collisions with obstacles has been used to learn the proposed ANFIS navigation algorithm. The Four- wheel autonomous robot is able to avoid the dynamic obstacle using the kinect camera. The proprietary software running on the Kinect provides full-body, three-dimensional, motion capture, facial and voice recognition capabilities and cheap. Comparisons between AI and different conventional techniques show the effectiveness of the ANFIS algorithm technique for navigation control of wheeled autonomous robots both in simulation and experimental mode. It is observed that the autonomous robot is able to reach the target efficiently during simulation and experiment. However, there is an error ratio of the length of the 
path of $7 . \vee \vee \%$ and an error rate of the arrival time of the target of $\vee . r 0 \%$ between simulation and experiment because of the friction between mechanical parts and friction with the ground. The experimental result shows that using ANFIS and machine vision as visual feedback makes the autonomous robot able to follow a smooth and efficient path avoiding collisions with the moving and stationary obstacles.

\section{$\checkmark \quad$ References}

['] H. Smith, "Software Development For High-Speed Autonomous Ground Vehicles," $r \cdot 19$.

[ץ] A. K. Rath, D. R. Parhi, H. C. Das, M. K. Muni, and P. B. Kumar, "Analysis and use of fuzzy intelligent technique for navigation of humanoid robot in

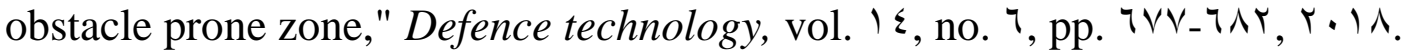

$\left[{ }^{\top}\right] \quad$ D. R. Parhi and P. K. Mohanty, "IWO-based adaptive neuro-fuzzy controller for mobile robot navigation in cluttered environments," The International Journal of Advanced Manufacturing Technology, vol. ^r, no. 9-1r, pp. 17.V-17ro, r.17.

[〔] H. Rawat, D. R. Parhi, B. Priyadarshi, K. Pandey, and A. K. Behera, "Analysis and Investigation of Mamdani Fuzzy for Control and Navigation of Mobile Robot and Exploration of Different AI Techniques Pertaining to Robot Navigation," Emerging trends in Engineering, Science and

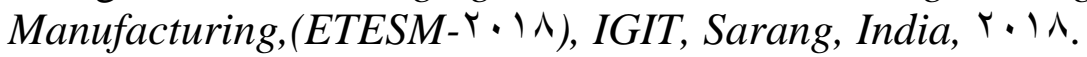

${ }^{\circ}{ }^{\circ} \quad$ A. Pandey and D. R. Parhi, "New algorithm for behaviour-based mobile robot navigation in cluttered environment using neural network architecture," World Journal of Engineering, vol. $1 r$, no. $r$, pp. $\left|r q_{-}\right| \leqslant 1, r_{.} \mid 7$.

[?] T. T. Van Nguyen, M. D. Phung, and Q. V. Tran, "Behavior-based navigation of mobile robot in unknown environments using fuzzy logic and multi-objective

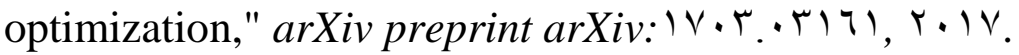

$[\vee] \quad$ O. R. E. Motlagh, T. S. Hong, and N. Ismail, "Development of a new minimum avoidance system for a behavior-based mobile robot," Fuzzy Sets and Systems,

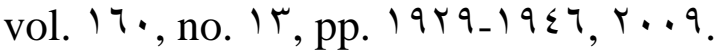

[^] P. K. Mohanty and D. R. Parhi, "A new hybrid intelligent path planner for mobile robot navigation based on adaptive neuro-fuzzy inference system," Australian Journal of Mechanical Engineering, vol. ' $r$, no. $r$, pp. 190-r. $\vee$, Y.10.

[9] D. Parhi and M. Singh, "Navigational path analysis of mobile robots using an adaptive neuro-fuzzy inference system controller in a dynamic environment," Proceedings of the Institution of Mechanical Engineers, Part C: Journal of

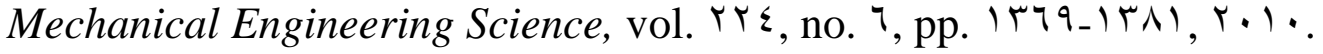


[1·] H. Chen, H. a. Yang, X. Wang, and T. Zhang, "Formation control for car-like mobile robots using front-wheel driving and steering," International Journal of

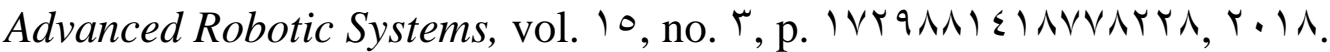

[1'] X. Wu, M. Xu, and L. Wang, "Differential speed steering control for fourwheel independent driving electric vehicle," in r.1r IEEE International Symposium on Industrial Electronics, $\uparrow \cdot 1 r$ : IEEE, pp. I- 7.

$\left[{ }^{Y}\right] \quad$ M. H. Achmad, N. A. Murtdza, N. A. A. Lokman, M. R. Daud, S. Razali, and D. Pebrianti, "Exploration of Unknown Environment with Ackerman Mobile Robot using Robot Operating System (ROS)," in Joint International Conferences on Electrical, Control and Computer Engineering, r.10, pp. IVOVT-IVOVa.

[1ץ] X. Bajrami, A. Dërmaku, N. Demaku," Artificial Neural Fuzzy Logic

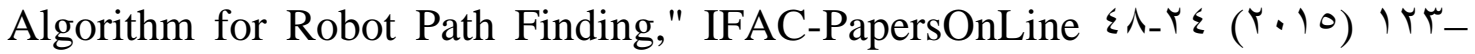
ITY.

[1ミ] A. D. Olaru, S. A. Olaru, N. F. Mihai, and N. M. Smidova, "Animation in Robotics with LabVIEW Instrumentation," International Journal of Modeling and Optimization, vol. ${ }^{9}$, no. ',$~ r .19$.

[10] M. A. Olivares-Mendez, S. Kannan, and H. Voos, "Vision based fuzzy control autonomous landing with UAVs: From V-REP to real experiments," in $r \cdot 10$ rrrd Mediterranean Conference on Control and Automation (MED), r.10: IEEE, pp. $\mid \leq-r)$. 